\title{
Pricing of uncertain certified emission reductions in a Chinese coal mine methane project with an extended Rubinstein-Ståhl model
}

\author{
Xiangyang Xu • Joanna Horabik • Zbigniew Nahorski
}

Received: 2 January 2013 / Accepted: 5 January 2014 / Published online: 1 March 2014

C The Author(s) 2014. This article is published with open access at Springerlink.com

\begin{abstract}
The development of coal mine methane (CMM) projects is subject to various kinds of risk, one of these being their highly variable methane content. In this study, a new methodology is proposed to reflect the impact of this uncertainty on a negotiated Certified Emission Reduction (CER) price, which is based on the available information. To simulate a process of price negotiation the Rubinstein-Ståhl bargaining game is utilized, where a buyer's discount factor is unknown. It is assumed that a buyer's willingness to accomplish price negotiations depends on the CER uncertainty. The bargaining model has been extended by introducing dependence of its three parameters on the probability of a failure to fulfil the contracted CER amount. To quantify this probability, we develop a conditional distribution given information on the point estimate of methane amount for the project under consideration, and on the distribution of available estimates from coal mines having similar characteristics. The proposed approach is applied to a particular CMM capture and utilization project in Anhui province, China. The results indicate that the uncertainty influence is significant, particularly when the credibility of a seller increases, i.e. the probability of a failure to fulfil the project decreases. The analysis can be of use to both negotiating parties at an early stage of a comprehensive CMM project planning.
\end{abstract}

\section{Introduction}

The Clean Development Mechanism (CDM) has been introduced as one of the three flexible mitigation mechanisms in the Kyoto Protocol. The CDM allows developed countries listed in Annex 1 of the UN Framework Convention on Climate Change (UNFCCC) to invest in

This article is part of a Special Issue on "Third International Workshop on Uncertainty in Greenhouse Gas Inventories" edited by Jean Ometto and Rostyslav Bun.

Electronic supplementary material The online version of this article (doi:10.1007/s10584-014-1057-8) contains supplementary material, which is available to authorized users.

X. Xu

China University of Mining and Technology (Beijing), D11 Xueyuan Road, Haidian District, Beijing 100083, China

J. Horabik $(\bowtie) \cdot$ Z. Nahorski

Systems Research Institute, Polish Academy of Sciences, Newelska 6, 01447 Warsaw, Poland

e-mail: Joanna.Horabik@ibspan.waw.pl 
greenhouse gas (GHG) emission reduction projects in non-Annex 1 developing countries. It enables Annex 1 countries to offset this part of their emissions reduction commitments, and the host developing countries gain in return from the technology and financing necessary for GHG prevention.

The CDM market is dominated by China. By the 1st of March 2011, over 1,200 Chinese CDM projects had been registered, accounting for $43.25 \%$ of the total number globally. The estimated annual Certified Emission Reductions (CERs) from these projects account for $62.63 \%$ of the total CERs from all remaining CDM projects (UNFCCC 2011).

Among the above-mentioned Chinese CDM projects, there are 42 coal bed methane (CBM) and coal mine methane (CMM) projects. In $\mathrm{CBM}$, methane is drawn to the surface, and the exploitation process is similar to that of natural gas. In CMM, methane is extracted during underground coal mining operations. The introduction of advanced technologies enables it to be used as a fuel, see e.g. Utaki (2010), which may be approved as a GHG Certified Emission Reduction. Otherwise, the methane discharged from coal seams is usually emitted into the atmosphere. The reported annual CERs from the mentioned Chinese methane CDM projects amount to over 16 million $\mathrm{tCO}_{2}$ eq, as of the 1st March 2011 (UNFCCC 2011). Recovery and usage of CMM resources is enthusiastically promoted by the Chinese government for CDM projects.

The methane global warming potential (GWP) is $21^{1}$ times higher than that of carbon dioxide (IPCC 2007). Effective methods to restrict methane emissions from a variety of sources are investigated in e.g. Brown et al. (2010); Magalhães et al. (2010); Oh et al. (2010). CMM projects provide one more opportunity to reduce methane emissions.

Compared with other CDM mechanisms, the number of registered CMM projects is relatively low. Among others, one of the recognized reasons for this is the fact that the methane content of CMM differs substantially among projects; this may even be the case for one particular project. The quantification of the CER amount from a CMM project is based upon estimates of carbon emissions from coal resources. Numerous factors underlie these estimations. Firstly, the geological conditions of a coal mine: the methane content in coal resources, methane quality and stability, which is of great importance for the final-users, as well as coal methane reserves. The coal methane reserve factor includes the thickness of a coal seam, the depth of the deposit, permeability (Zhang et al. 2004) as well as the reserve pressure (Shimada et al. 2005). Secondly, the technology employed in mining coal methane resources matters (Zhang et al. 2005; Xu 2007a). An estimation of the amount of CER is conducted as a result of expert knowledge of the geological conditions and technology available. However, this is a challenging task. For the vast majority of inaccurate estimations, CER amounts tend to be overestimated.

In this paper, we analyse the influence of an uncertainty of CER amount on its price. Specifically, we focus on the uncertainty that is related to an imprecise knowledge of the methane content in a coal bed. Thus far, this kind of uncertainty has not been formally taken into account in price negotiations, nor in the Kyoto Protocol compliance condition of buyers. It is, however, reasonable to assume that an uncertain amount of CER has an influence on the buyer's decision. While an inclusion of this uncertainty in the buyer's compliance can be solved by adaptation of the methodology proposed in Nahorski et al. (2007), and by that shortly to be presented in Nahorski et al. (2014), the influence of uncertainty on the price negotiation process has not been previously analysed.

\footnotetext{
${ }^{1}$ According to the IPCC 2nd assessment report, relevant for emissions reporting under Kyoto Protocol. The 4th assessment report (IPCC 2007) gives a GWP of 25 and is likely to be taken on board in future.
} 
To start a CDM project, a buyer and a seller have to sign the Emission Reduction Purchase Agreement (ERPA), which is the contract underlying the trading of CERs. For this, a unit price of CERs is of great importance. In Chinese CDM projects, the CER price usually has to be bargained at the initial point of negotiations. In this study, to simulate a price negotiation process, we utilize the two-player Rubinstein-Ståhl bargaining game of alternating offers.

The Rubinstein-Ståhl game has been applied to model practical bargaining settings, such as labor negotiation (Vannetelbosch 1997) or conflict resolution in the conjunctive use of surface and groundwater resources (Kerachian et al. 2010). It provides a suitable tool to model CER pricing, since impatient bargaining plays an important role in this kind of negotiations. Buyers, being usually international or governmental agencies, are not willing to put much effort into negotiating a single project. Typically, if after one or two rounds the parties cannot reach an agreement, the buyer gives up since they has many other opportunities for investment. For instance, Hovi (2001) considered the Rubinstein-Ståhl bargaining scheme while modelling the issue of international bargaining and enforcement, and for this the CDM was used as an example.

The main idea of our proposition is that for highly uncertain CERs, buyers tend to offer lower unit prices. Starting from this assumption, we propose to reflect this uncertainty in the parameters of the incomplete information case of the Rubinstein-Ståhl bargaining game (Rubinstein 1985), hereafter called the extended Rubinstein-Ståhl bargaining game. The functional dependence of the parameters upon the uncertainty is proposed. To quantify the uncertainty of the amount of CER, two technical indices of methane content are used independently. Each of them provides information on a point estimate of the amount of methane and on the distribution of its uncertainty. These two types of information are combined by means of the probability theory. The results of the bargaining process are compared for the two methane content indices, for the modified bargaining parameters, and with respect to the introduced probability parameter $\alpha$ of being unable to fulfil the contracted amount of CER.

The methodology is exemplified by a particular CMM capture and utilization project in Anhui province, China. Nevertheless, the proposed approach is general in nature, and it can be applied for modelling the influence of the uncertainty of other estimated variables, subject to relevant modifications.

\section{Methodology}

The proposed approach for the pricing of uncertain CERs with the Rubinstein-Ståhl includes several stages. We propose to express the uncertainty underlying the estimation of the amount of CER as a conditional distribution given information on a point estimate of the amount of methane in the project under consideration and on a distribution of available estimates from similar coal mines. Furthermore, the layout of the bargaining game is extended by introducing the uncertainty in two parameters: (i) discount factor reflecting buyer's patience and (ii) the probability that the buyer is weak. The following sections describe the main components of the methodology.

\subsection{The Rubinstein-Ståhl bargaining model}

The Rubinstein- Ståhl game simulates a negotiation process where two players: a buyer and a seller, denoted with the superscripts ${ }^{B}$ and ${ }^{S}$, respectively, bargain to share a surplus of size $k$. Starting at an initial period $t=0$, each player makes a proposal in turn as to how to divide the surplus, and the other player may agree to the offer or reject it. The acceptance of the offer terminates the bargaining process. On the other hand, a rejection means that the players enter 
the next negotiation period $t+1$, in which the roles of the two players are reversed and the side who had rejected the first offer makes a counter offer, as illustrated in Fig. 1.

Both players discount the future at a constant rate. The discount factors $\delta^{i}, i=B, S$ reflect the players' impatience - the closer they approach 1, the greater the players' patience.

Rubinstein (1982) used the concept of the subgame perfect equilibrium strategy to solve the negotiating problem for an infinite horizon in the case of complete information. Complete information means that the preference relations of both players are common knowledge. For the fixed discounting factors Rubinstein (1982) showed that a unique pair of bargaining strategies exist, specifying the best response to each player's offer at every point in time. In the equilibrium, the surplus $k$ is divided between the player making the initial offer, $I$, and the one following, $F$, as $\frac{1-\delta^{I}}{1-\delta^{I} \delta^{F}}$ and $\frac{\delta^{I}\left(1-\delta^{F}\right)}{1-\delta^{I} \delta^{F}}$, respectively.

Unlike the original exposition of the Rubinstein (1982) game, with the commonly known discount factors of both players, in this paper we consider a somewhat more realistic version of the scheme with incomplete information. Assume that both players know the seller's discount factor $\delta^{S}$, but the buyer's discount factor $\delta^{B}$ remains undisclosed. A buyer can be one of two types: weak (i.e. impatient), with a lower discount factor $\delta_{l}$ or strong (i.e. patient), with a higher discount factor $\delta_{h}$, and it holds $0<\delta_{l}<\delta_{h}<1$. To simplify the notation we drop the superscript ${ }^{B}$ denoting the buyer's discount factor, both above and in the following. A seller needs to assess the probability $p$ that the buyer is weak. It may be concluded from the buyer's behavior; although a buyer may try to pretend that they are stronger than they actually are.

Bargaining with one-sided uncertainty has been studied by Rubinstein (1985) and reexamined by Bikhchandani (1992), see also Srivastava (2001) for an experimental testing. Rubinstein (1985) showed that a unique bargaining sequential equilibrium exists and takes the form of the following theorem. For a game starting with the seller's offer:

(i) If $p$ is high enough so that $y^{p}>\delta^{S} V_{h}$, then a seller offers $x^{p}$. The weak buyer accepts this offer, while the strong buyer rejects it and offers $y^{p} \leq x^{p}$, which is accepted. Both $x^{p}$ and $y^{p}$ denote the seller's share.

(ii) If $p$ is low enough so that $y^{p}<\delta^{S} V_{h}$, then a seller offers $V_{h}$ and both the weak and strong buyer accepts it.

Here,

$$
\begin{gathered}
V_{h}=\frac{1-\delta_{h}}{1-\delta^{S} \delta_{h}}, \\
x^{p}=\frac{\left(1-\delta_{l}\right)\left(1-\left(\delta^{S}\right)^{2}(1-p)\right)}{1-\left(\delta^{S}\right)^{2}+\delta^{S}\left(\delta^{S}-\delta_{l}\right) p}
\end{gathered}
$$

and

$$
y^{p}=\frac{\delta^{S}\left(1-\delta_{l}\right) p}{1-\left(\delta^{S}\right)^{2}+\delta^{S}\left(\delta^{S}-\delta_{l}\right) p} .
$$

The theorem states that the equilibrium is reached not later than the second period. The value $V_{h}$ is equal to the seller's share in the complete-information-game equilibrium where the seller starts bargaining and the buyer is a strong one. Thus, for low $p$, the weak buyer is better off than in the complete-information case. For high $p$, both $x^{p}$ and $y^{p}$ increase in $p$. This is intuitive, because the more likely the buyer is weak, the more favourable the situation is for the seller. 


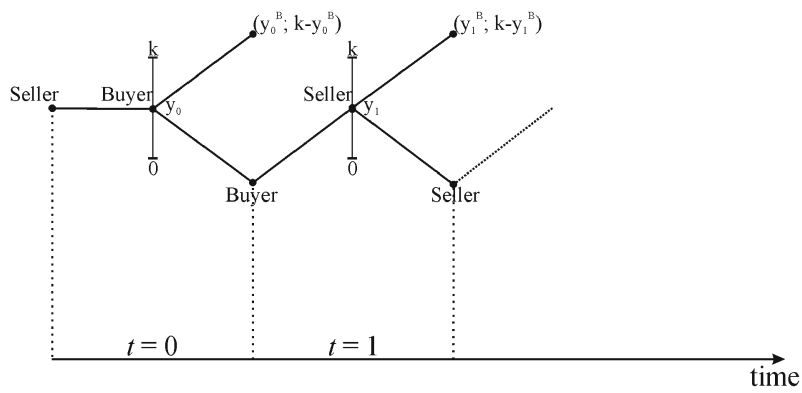

Fig. 1 Scheme of bargaining game with alternating offers

For the convenience of calculation, we can determine the boundary point $p^{*}$ as that at which $y^{p}=\delta^{S} V_{h}$

$$
p^{*}=\frac{\delta^{S}+1}{\delta^{S}+\frac{1-\delta_{l}}{1-\delta_{h}}}
$$

If $p>p^{*}$ case (i) holds.

\subsection{Modelling uncertainty of the methane content distribution}

Various kinds of risk influence the CER price negotiations. For the methane CDM projects these include: selection of a project site, supply and demand constraints on the electricity market in neighboring regions, potential difficulties with CER approval, validation, registration, monitoring or certification.

Among other risks, the uncertainty of estimating the amount of CER is of special importance for the methane related CDM projects. Usually only a point estimate of the methane amount is provided, denoted as $\widehat{x}$. However, this amount is uncertain and the real value may differ. The uncertainty will be expressed by a conditional probability distribution $f(x \mid I)$, where $I$ stands for our prior knowledge. In this study, it is assumed that a priori we know the probability distribution function $g(x)$ of the uncertain amount of CER with its variance $\sigma^{2}$, and the estimate $\widehat{x}$. The lognormal distribution is adopted, in accordance with the statistical inference described in Subsection 3.3, i.e.

$$
g(x)=\frac{1}{\sqrt{2 \pi} \sigma x} \exp \left[-\frac{(\ln x-\mu)^{2}}{2 \sigma^{2}}\right], \quad x>0 .
$$

Its mean is $E(X)=\exp \left(\frac{1}{2} \sigma^{2}+\mu\right)$, median $m=\exp (\mu)$, and the variance is $\operatorname{Var}(X)=$ $\exp \left(\sigma^{2}+2 \mu\right)\left(\exp \sigma^{2}-1\right)$. We use this distribution to infer on the uncertainty of the observation $\widehat{x}$. In the situation under consideration, the value $\widehat{x}$ actually adds information to our knowledge of the prior distribution $g(x)$. We make the assumption about the parameter $\mu$, that it belongs to a given set $M$, i.e. $\mu \in M$. Now, using the law of total probability we have

$$
f(x \mid g, \widehat{x})=\int_{M} g(x \mid \mu) \pi(\mu \mid \widehat{x}) d \mu
$$


where $\pi(\mu \mid \widehat{x})$ is the conditional probability density function of $\mu$ when $\widehat{x}$ is known. However, we know that, when $\widehat{x}$ has been observed, both $\widehat{x}$ and $\mu$ are deterministic values. Thus, $\pi(\mu \mid \widehat{x})$ is simply a deterministic function $\mu(\widehat{x})$. Formally, we insert $\pi(\mu \mid \widehat{x})=\delta(\mu-\mu(\widehat{x}))$ into (5), where $\delta$ is the Dirac delta function and $\mu(\widehat{x})$ is a deterministic value. This gives $f(x \mid g, \widehat{x})=g(x \mid \mu(\widehat{x}))$.

To find the function $\mu(\widehat{x})$ we estimate $\mu$ in (4) using the maximum likelihood method with one observation $\hat{x}$. Consequently, we have

$$
\ln g(x \mid \mu)=-\ln \sqrt{2 \pi} \sigma-\ln x-\frac{1}{2 \sigma^{2}}(\ln x-\mu)^{2} .
$$

The maximum of this function with respect to $\mu$ is for

$$
\mu(\widehat{x})=\ln \widehat{x}
$$

Inserting this value into (4) we finally get

$$
f(x \mid g, \hat{x})=\frac{1}{\sqrt{2 \pi} \sigma x} \exp \left[-\frac{\left(\ln \frac{x}{\hat{x}}\right)^{2}}{2 \sigma^{2}}\right], \quad x>0 .
$$

As $\widehat{x}=e^{\mu}$, then $\widehat{x}$ is the median of the above distribution.

Although $\hat{x}$ seems to be a natural value to be taken as the amount of CER, the question arises as to whether another value might be more profitable from the seller's point of view. Let us introduce the probability $\alpha \in[0,1]$ and denote by $x_{\alpha}$ the quantile of order $\alpha$ of the distribution, compare also Fig. 2. The true value of the amount of methane is lower than $x_{\alpha}$ with probability $\alpha$. In other words, $\alpha$ expresses the probability that the terms of the CMM project, with the amount of CER equal to $x_{\alpha}$, may not be fulfilled due to an insufficient amount of methane. To avoid reference to specific values, in the following we use the ratio $r(\alpha)=\frac{x_{\alpha}}{\widehat{x}}$, which is a non-dimensional value.

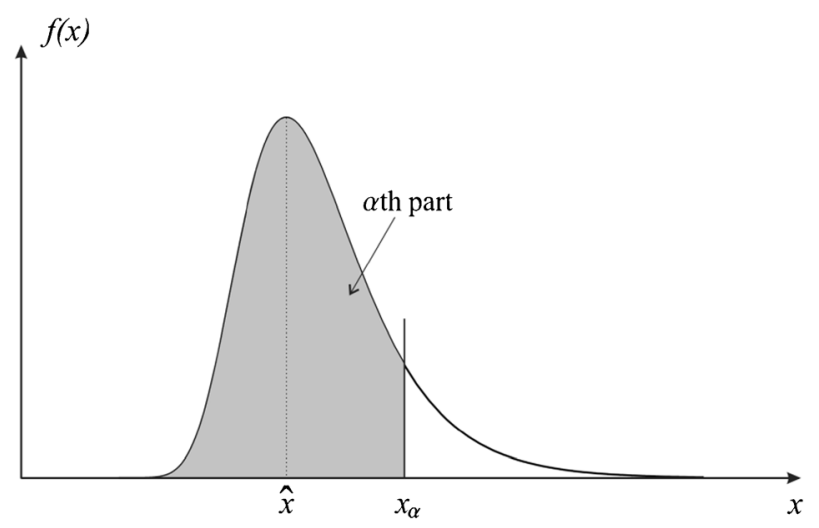

Fig. 2 A distribution of uncertainty underlying methane calculations $f(x)$, a calculated methane amount $\widehat{x}$, and an amount of methane $x_{\alpha}$ corresponding to the probability $\alpha$ 
Since the function $f(x \mid g, \widehat{x})$ follows the lognormal distribution, its cumulative distribution function (cdf) is

$$
F\left(x_{\alpha}\right)=\varphi\left(\frac{\ln x_{\alpha}-\mu}{\sigma}\right)=\alpha,
$$

where $\varphi$ is cdf of the standard normal distribution. We obtain

$$
x_{\alpha}=\exp \left[\mu+\sigma \varphi^{-1}(\alpha)\right] .
$$

In our case $\mu=\ln \widehat{x}$, therefore it follows

$$
r(\alpha)=\frac{x_{\alpha}}{\widehat{x}}=\frac{\exp \left[\mu+\sigma \varphi^{-1}(\alpha)\right]}{\widehat{x}}=\exp \left[\sigma \varphi^{-1}(\alpha)\right],
$$

and $r(\alpha)$ does not depend on $\widehat{x}$.

\subsection{Modelling beliefs on parameters of bargaining model}

Any change of the amount of CER is to the detriment of both parties. Due to the highly attractive commercial benefits of CDM projects, a project buyer is in pursuit of high volumes of CERs. Obviously, the higher the CER amount is, the more valuable a project becomes. However, if the amount of methane actually generated is lower than the amount considered in a negotiation period, it affects the buyer's benefit, due to the favourable conditions a buyer might have given to a seller during the negotiations.

In this subsection, we aim to incorporate the risk into the extended Rubinstein-Ståhl negotiation model, so that the seller's belief on a buyer's negotiation position reflects the uncertainty of calculating the amount of methane. It is assumed that the uncertainty of assessing the amount of CER is known to the buyer. So, both the estimates of the buyer's discount rates $\left(\delta_{l}, \delta_{h}\right)$ and the probability $p$ are affected by this risk, which we express with respective functional dependences. For this, a set of common sense assumptions are taken and possibly the simplest relationships that satisfy them are constructed.

Discount factors $\delta_{l}, \delta_{h}$. Let us denote the lower and upper estimates of a low discount factor $\delta_{l}$ by $\delta_{l l}$ and $\delta_{l h}$, respectively. Similarly, let $\delta_{h l}$ and $\delta_{h h}$ stand for the lower and upper estimates of a high discount factor $\delta_{h}$. Assuming a linear positive relationship between the uncertainty $\alpha$ and buyer's unknown discount factors $\delta_{l}$ and $\delta_{h}$

$$
\begin{gathered}
\delta_{l}(\alpha)=\delta_{l l}-\alpha\left(\delta_{l l}-\delta_{l h}\right) \\
\delta_{h}(\alpha)=\delta_{h l}-\alpha\left(\delta_{h l}-\delta_{h h}\right) .
\end{gathered}
$$

The above expressions approximate the main relations in the simplest way. Greater uncertainty $\alpha$ of the CER amount estimation strengthens the buyer's position, and this is reflected in their higher discount factors.

Probability $p$. We model the probability $p$ that the buyer is weak as a third order polynomial of $\alpha$. It is the simplest polynomial satisfying the following conditions

$$
p(0)=1 \quad p(1)=0,
$$


which are assumed to be approached slowly

$$
p^{\prime}(0)=p^{\prime}(1)=0,
$$

where $p^{\prime}(\cdot)$ denotes the first derivative. The conditions provide the following function $p(\alpha)$

$$
p(\alpha)=2 \alpha^{3}-3 \alpha^{2}+1, \quad \alpha \in[0,1] .
$$

The function is monotonically decreasing, expressing the intuition that the higher the uncertainty of methane calculations is, the stronger is the buyer's position, and thus the lower is the probability that they are weak.

\section{Case study}

\subsection{Introductory information on the Huainan project}

The considered CMM capture and utilization project is located in Huainan, Anhui Province of East China. The coal mine belongs to Huainan Coal Mining Group Co., Ltd., situated in the north central part of Anhui Province. Although coal resources of the Huainan mining area are very rich, the geological conditions are complex. Many of the mines experience a high content of explosive mine gas, largely composed of methane and, thus, extremely hazardous to miners.

Within the initiated project, installed technologies capture the CMM and enable its use as a fuel source. This prevents methane from being released into the atmosphere, and increases the safety of coal extraction at the same time. As a result, emission reductions were achieved through combustion of the extracted CMM, which replaced conventional coal usage in more than 15,000 households and was also used in the generation of electricity in the industry sector (Project Document Description 2006).

As mentioned before, division of the margin between the buyer's highest CER unit price and the seller's (Huainan Coal Mining Group Co., Ltd.) lowest price is negotiated at the ERPA stage. In the following we assume that a broker function is incorporated into either the buyer's or seller's side.

\subsection{Negotiating CER price: no uncertainty case}

In this subsection we consider the negotiation of the CER price, taking no account of the uncertainty of the methane content. To apply the bargaining theory, proper discount factors should be selected for both parties. In practice, this becomes a challenging task. This is particularly true for a buyer's discount rate, which remains basically unknown.

In this case study, to set the values of discount factors (or, in the following, their upper and lower limits) expert assessments were used that were based on the project's documentation. Below we list the most important factors influencing the negotiation positions of the CMM project parties. First of all, there is the costs of communication between parties. Usually English is the working language, so a seller (the Chinese side) needs to hire some interpreters. On the other hand, a buyer also needs to hire some local experts or establish an agency in China to facilitate negotiations. Secondly, collating information comes at a cost for both buyer and seller and acts as a further drain on their discount rates. Before a buyer gives an initial price, they must verify the information on the project from various channels; not only those limited to formal documents and local information, but also from the authorized third parties. 
In the current conditions of the Chinese CDM market, the sellers are more concerned to sell the CERs than the buyers are to buy them. This is due to the numerous other opportunities available to the buyers to find a more profitable option. Big international buyers usually possess extensive knowledge of the international CDM market, which besides China, also includes India, South America and others.

Obviously, both sides hope to reach an agreement (i.e. sign ERPA) as soon as possible. The buyer's patience is closely related to the international carbon market price and its domestic environmental policy. That is, when the international carbon market price is decreasing, a buyer will have a lower discount factor. On the other hand, the Chinese side is usually unfamiliar with the international carbon market along with environmental policy of a buyer's country. Therefore, from the seller's point of view, the buyer's discount rate is assumed to be unknown. The seller's discount factor is mainly decided upon by the project's risk factor.

Here, the seller estimates that the buyer is either a weak player with a discount rate $\delta_{l}=0.91$, or that they are a strong player with a discount rate $\delta_{h}=0.975$. Furthermore, the seller assumes that these two situations have equal probabilities, i.e. $p=0.5$. The seller's discount factor $\delta^{S}=0.94$ remains common knowledge.

For a case with no account of uncertainty, we analyse a real bargaining situation from the past, and therefore use the costs and prices from that period of time. The aim is to check whether the prices calculated from the model are comparable with the actual negotiated ones. When the Huainan project was negotiated, the floor CER price demanded by the Chinese authorities for CMM projects was set at 8 Euro (approximately 70 Yuan/ton); we treat this value as the seller's conservative price. The buyer set his conservative price at 170 Yuan/ton, which yielded a surplus $k=100$ Yuan/ton to be divided among the players.

Negotiations started with the seller's offer. From (3) we calculate $p^{*}=0.45$, and since $p>p^{*}$, we follow case (i) of the Theorem. The seller proposed that their benefit is

$$
k * x^{p}=k \frac{\left(1-\delta_{l}\right)\left(1-\left(\delta^{S}\right)^{2}(1-p)\right)}{1-\left(\delta^{S}\right)^{2}+\delta^{S}\left(\delta^{S}-\delta_{l}\right) p}=38.49 \quad(\text { Yuan } / \text { ton })
$$

from where the CER price is 108.49 (Yuan/ton). The weak buyer accepted this offer, whereas the strong one rejected it. Then, in the next step, the strong buyer offered to the seller a benefit of

$$
\left.k * y^{p}=k \frac{\delta^{S}\left(1-\delta_{l}\right) p}{1-\left(\delta^{S}\right)^{2}+\delta^{S}\left(\delta^{S}-\delta_{l}\right) p}=32.41 \quad \text { (Yuan } / \text { ton }\right)
$$

yielding the CER price of 102.41 (Yuan/ton). Comparing these results to the CER price negotiated for the Huainan project, the actual buyer was a weak one.

\subsection{Estimating uncertainty of methane content assessment}

High risk of CER estimation in CMM projects is related to the selection of the project site. It determines such factors as methane reserves, geological mining conditions, gas quality, local demand, gas prices on the local market or currency exchange rate, etc. (Xu 2007b). These uncertainties and risks, although important for both the seller and buyer, are not considered in the study. Here we assume that the project site is known and we focus on an uncertainty of CER originating from inaccuracies in the assessment of the amount of methane for an already selected project site. 
For CER assessment, typically the ACM0008 methodology is applied, see ACM (2010) and UNECE (2010). According to this methodology, the annual (Certified) Emission Reductions $E R$ of a CDM project are calculated as

$$
E R=B E-P E-L E
$$

where $B E$ are the baseline emissions, saved due to the implementation of the project; $P E$ are the project emissions; and $L E$ are the leakage emissions. In the CMM case study under consideration, the baseline emissions are represented by the mined methane, which replaces the use of other fuels. Uncertainty of emission reduction is mainly due to baseline emissions $B E$, since project emissions $P E$ can be estimated with much better accuracy and leakage emissions $L E$ are minor. Therefore, the two latter sources of uncertainties are neglected, and it is assumed that uncertainty of the overall emission reduction is caused by the baseline emissions, which is strictly related to the methane content in the coal bed.

For the Huainan project, uncertainty of methane emission estimation is characterized with two indices: the highest methane content in the coal bed seam and relative methane emission, both measured in $\mathrm{m}^{3} / \mathrm{t}$. The former index is typically used in China to estimate future CER production, while the latter accounts for the technology that has been adopted and provides a more accurate estimation.

Figure 3 shows the positively skewed distributions of these two indices, based on data from 25 coal mines, which extract coal from beds of a similar nature in the region. Thus, the histograms show distributions of the indices in Chinese coal mines of the type similar to the one considered in the case study. We fit the lognormal distributions to the data for both indices, and test the results with the Kolmogorov-Smirnov test, see Table 1A of the supplementary material. Apart from the lognormal distributions, other density functions have also been considered (see the supplementary material). The lognormal distribution provided the best results out of the considered functions.

For the considered indices of the highest and relative methane content, the ratio $r(\alpha)$, calculated with Eq. (7), will be considered in the following. This is shown in Fig. 4b. We determine $r_{\text {min }}=r(\alpha=0)=0$, and due to the fact that the support of lognormal distribution is unbounded from above, we set $r_{\max }=r(\alpha=0.995)$, which provides $r_{\max }=2.79$ for the highest methane content and $r_{\max }=6.27$ for the relative methane content. Note that $r(\alpha=0.5)=1$, i.e. when $x_{\alpha}=\widehat{x}$. This is a consequence of the fact that $\widehat{x}$ is the median of the distribution $f(x \mid g, \widehat{x})$. The scale parameters have been estimated as $\sigma=0.399$ for the highest methane content and $\sigma=0.713$ for the relative methane content.

\section{Simulating negotiation outcome under uncertainty}

In this section, the influence of the uncertainty of methane calculations on the negotiated CER price is analyzed. The resulting CER prices are presented in terms of the seller's share of the surplus. To calculate a final CER unit price, a seller's share has to be multiplied by $k$ and added to their conservative price.

We begin with modelling the unknown buyer's discount factors $\delta_{l}$ and $\delta_{h}$, as well as the probability that a buyer is weak, see Fig. 4 . The following lower and upper limits of discount factors were assigned: $\delta_{l l}=0.88, \delta_{l h}=0.94, \delta_{h l}=0.96, \delta_{h h}=0.99$. Note that the previously assigned values $\delta_{l}$ and $\delta_{h}$ were fixed as the middles of the discount rates $\delta_{l l}, \delta_{l h}$ and $\delta_{h l}, \delta_{h h}$, respectively. As before, the seller's discount factor $\delta^{S}=0.94$ is adopted. 

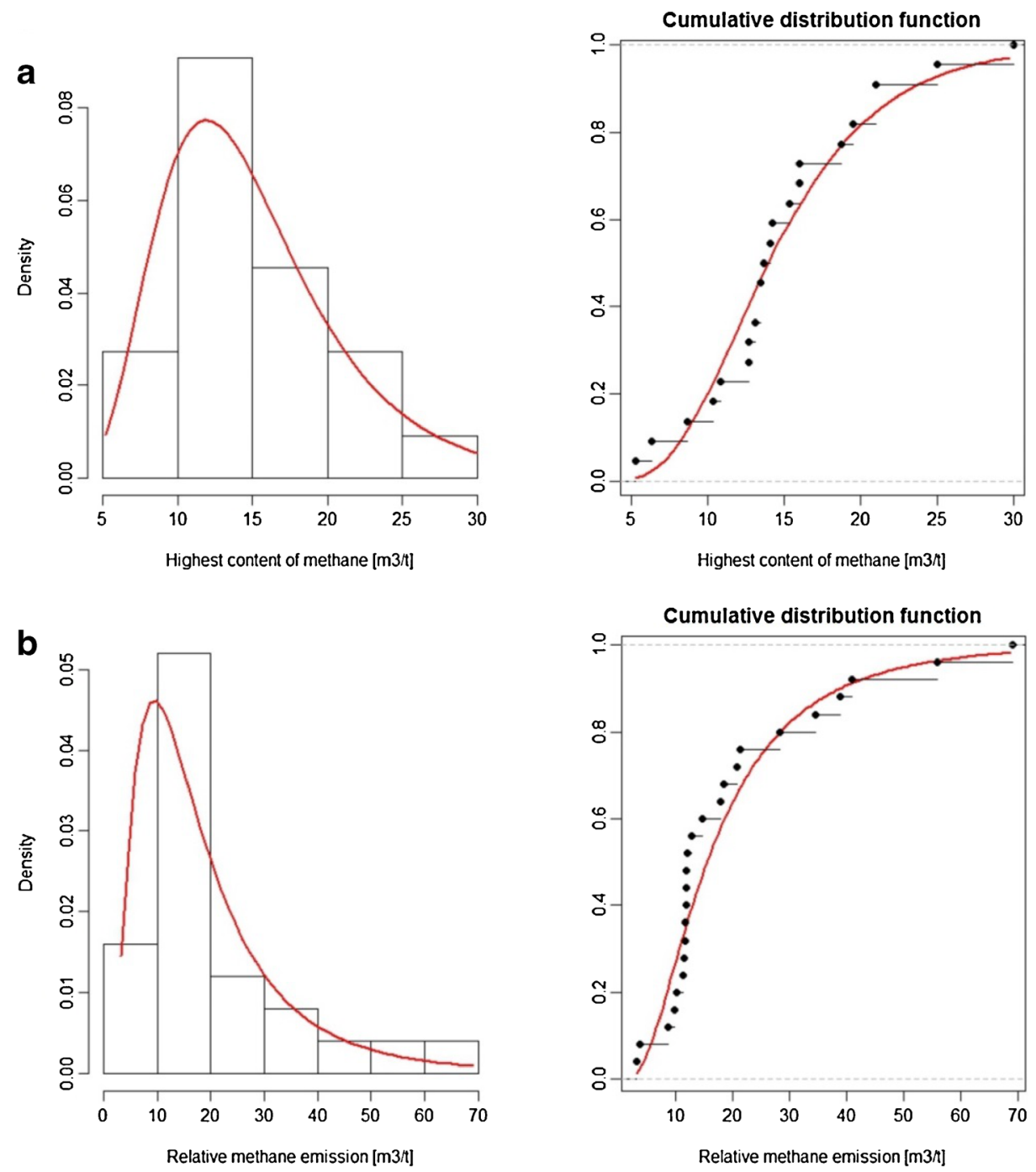

Fig. 3 Distributions of $\mathbf{a}$ highest methane content and $\mathbf{b}$ relative methane content with fitted lognormal density functions

Figure 4a depicts the boundary point $p^{*}=p$ distinguishing between the cases (i) and (ii) of the theorem (here, for $\alpha=0.56$ ), and Fig. $4 \mathrm{c}$ presents the optimal share of a surplus for a range of $\alpha$ values. The difference between the shares accepted by a strong and a weak buyer is about $5 \%$ of the surplus, and note that it is practically independent of $\alpha$ (as long as $\alpha<0.56$ ). Naturally, the higher the uncertainty $\alpha$ is, the lower share of a surplus the seller gets, and this value is decreasing from 0.69 down to 0.14 .

Figure $4 \mathrm{c}$ also provides comparison with the previously considered no-uncertainty case, depicted with red lines. For the values of discount factors under consideration, these two instances provide the same results for $\alpha=0.5$, as indicated with a dotted vertical line. Recall that $\alpha=0.5$ may be interpreted that the uncertainty is neglected, upon which the estimated value $x_{\alpha}=\widehat{x}$ is taken. There $r=1$, compare Fig. 4b. 
a

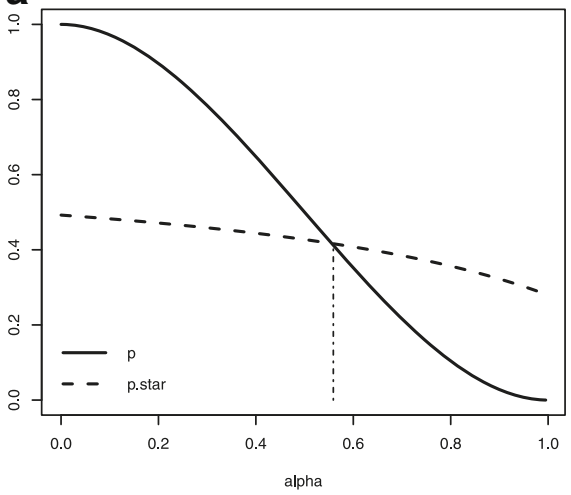

C

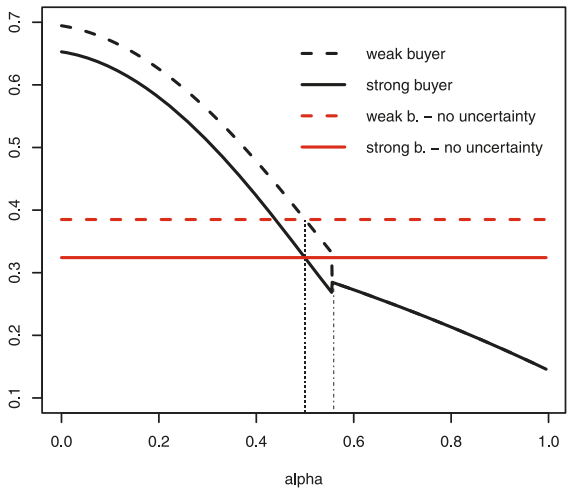

b

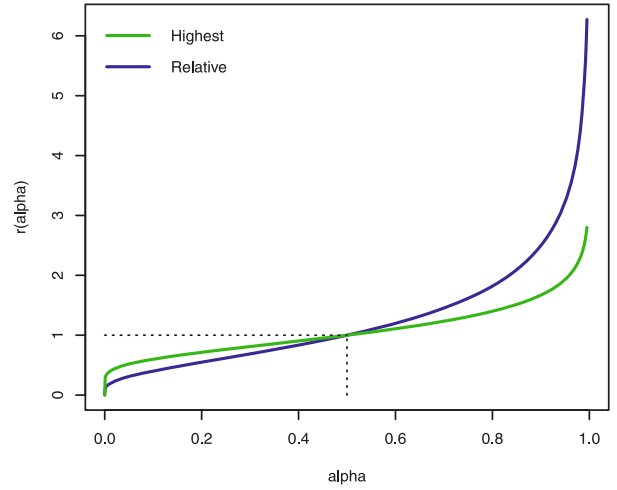

d

Seller's share

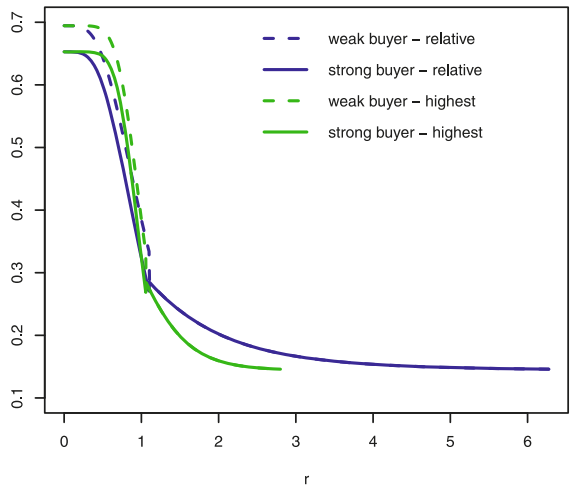

e

Seller's share

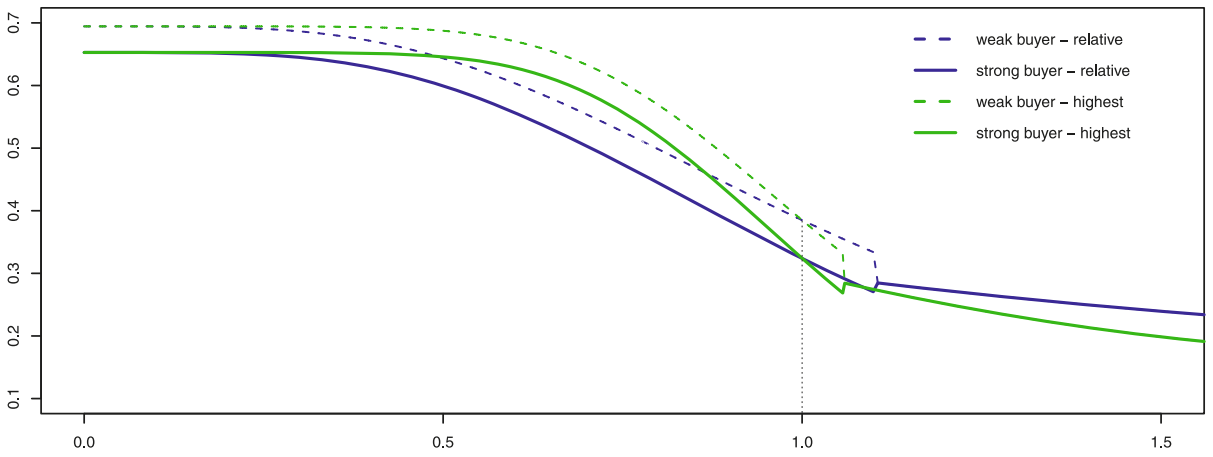

Fig. 4 Negotiation outcome: both $p$ and $\delta_{l}, \delta_{h}$ modelled

Figure $4 \mathrm{~d}$ depicts the negotiated share of a surplus as a function of the ratio $r$, illustrating the difference between two methane content indices. Figure 4e provides a more detailed version of the same graph, although for a limited range of $r$. As shown in this figure, as long as $r<1$, the seller is better off when the highest methane content index is considered, regardless of a buyer's type (weak or strong). This fact is in accordance with our intuition, because the 
uncertainty distribution of the highest methane content has a lower dispersion. It should be also noted that the highest methane content index is more often used in China. The situation changes when $r>1$. Then the reduction is less credible and it is better for the seller to consider the relative methane emission index. Technically, this is the effect of its higher dispersion. For a given value of $r>1$, the probability of not fulfilling the project is smaller for the relative methane emission index than for the highest methane content one. Overall, the consideration of the CER price in the function of $r$ expresses a seller's trade-off between gaining a better unit price which, however, requires assuring more credible emission reductions.

The proposed approaches for modelling discount factors $\delta_{h}$ and $\delta_{l}$, as well as for modelling probability $p$, do not need to be applied jointly. Each of these can be used as a separate part of an analysis. Figure 5 presents the negotiation outcome for the case of a constant $p=0.5$ (Fig. 5a), and for the case of a constant buyer's discount factors $\delta_{l}=0.91, \delta_{h}=0.975$ (Fig. $5 \mathrm{~b}$ ). In general, when either the discount factors or probability $p$ are modelled, the situation for a seller is less restrictive as compared with the previous results. In particular, for $\alpha$ close to 1, i.e. when almost the whole of the uncertainty distribution is accounted for, their share of a surplus does not drop below 0.24 , while it was 0.14 in the previous setting. Note that in the case of a constant $p=0.5$ (Fig. 5a), for the whole range of uncertainty parameter $\alpha$ the case (i) of the theorem applies, and the seller receives a different share depending on the type of the buyer.

a

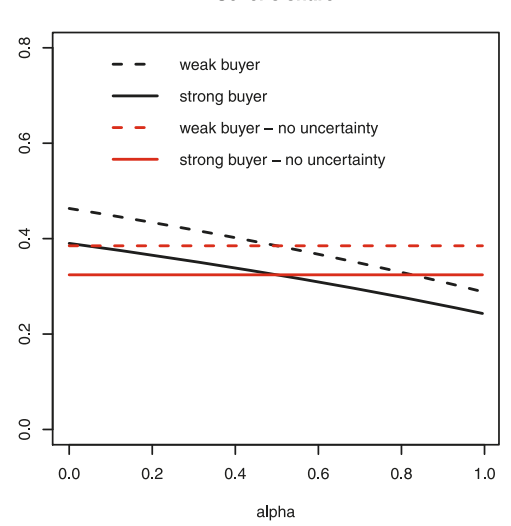

b

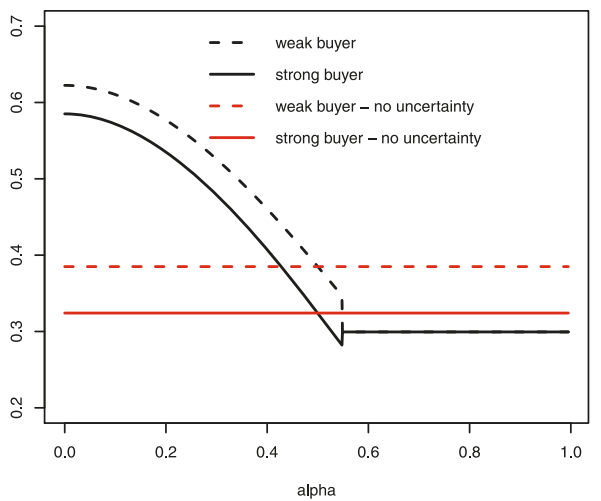

Seller's share

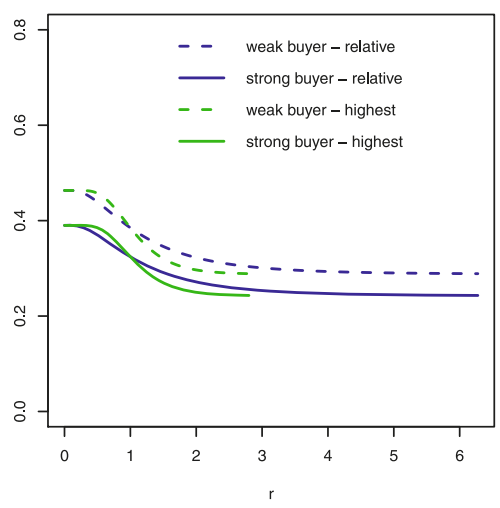

Seller's share

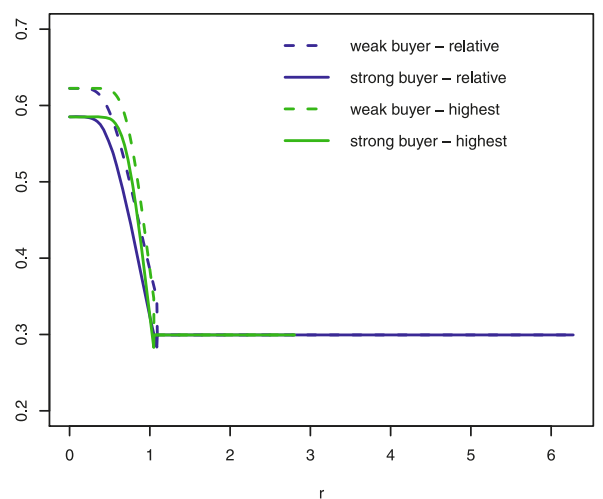

Fig. 5 Negotiation outcome for a constant $p=0.5$ and $\mathbf{b}$ constant $\delta_{l}=0.91, \delta_{h}=0.975$ 


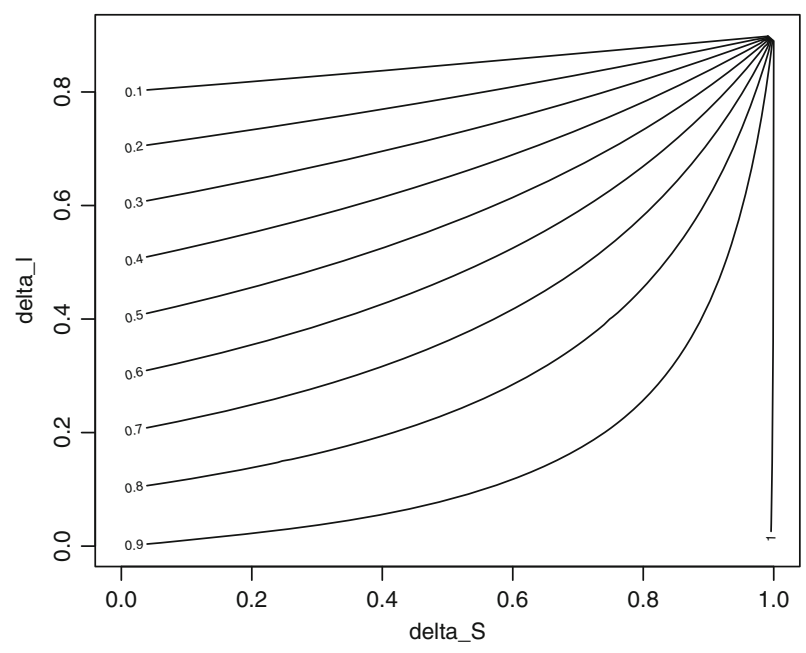

Fig. 6 Optimal seller's share for different discount factors; $p=0.5, \delta_{h}=\delta_{l}+0.1$

The applied Rubinstein-Ståhl bargaining game hinges upon assigned values of the discount factors. While the above analysis assumed a constant value of the seller's $\delta^{S}=0.94$, Fig. 6 presents the optimal seller's share for different values of discount factors for both negotiating parties. To this end, a simplified assumption was made $\delta_{h}=\delta_{l}+0.1$, and, furthermore, constant $p=0.5$ was assigned. The difference in results between the case of a weak and strong buyer was negligible, and therefore is not presented. The figure illustrates that for a high range of discount factors, e.g. higher than 0.7 , a negotiation outcome is highly sensitive to small changes in these values. This is not the case for a low range of discount factors.

\section{Summary and conclusions}

This paper addresses the problem of uncertainty in methane content for CMM projects, and its impact on a negotiated CER price. The Rubinstein-Ståhl bargaining model — in the case of incomplete information - is used to simulate negotiations concerning CER price. The bargaining model has been extended by introducing dependence of its three parameters on a distribution of methane content uncertainty, and more precisely on its quantile of order $\alpha$, where $\alpha$ is the probability of failure to fulfil the contract due to insufficient methane content in the coal bed. The lower and upper discount factors of a buyer have been chosen as simple linear functions in a predefined range of values. The probability that a buyer is a weak negotiator was designed as a third order polynomial, satisfying a few intuitive conditions.

The proposed methodology was applied for a CMM project implemented in the Huainan coal mine, the Anhui Province in Eastern China. Uncertainty distribution of the methane content was estimated using data on two types of indices, which were gathered from 25 Chinese coal mines with similar geological conditions.

The investigations revealed a few interesting results, fully compatible with those that were predicted as well as fitting in with current negotiation practise.

The uncertainty highly impacts the negotiation results and the parties' shares of the surplus. The dependence of the seller's share on the amount of methane adopted for the project is quite high, particularly for the values of $\alpha<0.5$ or $r<1$, that is for more credible methane contents. There, along 
with the decreasing probability of noncompliance the seller's share rises fairly rapidly. The gain of the seller's share, when negotiated with a weak buyer instead of a strong one is about $5 \%$ of the surplus, almost independent of the uncertainty parameter $\alpha$. This result indicates that a seller can earn a considerable amount of money by being presented with the chance to negotiate with a weak buyer.

Although the analysis covers the full range of the probability $0 \leq \alpha \leq 1$, it is rather unreasonable for the seller to consider either very small or very high values of $\alpha$. Still, the analysis of this paper presents only a partial answer to the choice of $\alpha$. Other aspects should also be taken into account, such as the gains achieved when the declared CER amount is satisfied as well as the costs of failing it. To solve these questions the CER price has to be known. In this respect, an analysis of the price bargaining, like that which is presented here, must be an essential part of a comprehensive project planning.

Acquisition of the discount factor values is a difficult part of the analysis. Intuition and experience as well as some intelligence methods may help to assign them. For instance, in their case study Kerachian et al. 2010 utilize the fuzzy set theory to better define uncertainty in utility functions, which, together with discount factors, define the preferences of parties. Another approach would be to introduce the learning stimuli in the bargaining strategies based on the information gathered, but this requires longer sequences of negotiation periods.

In this study simple models of the buyer's reaction to uncertainty have been assumed. More advanced modelling requires wider analysis of the buyer's incentives in the project, accounting also for other factors, such as the project's size.

Acknowledgements The study was conducted within: (i) the Joint China-Poland Intergovernmental Science and Technology Cooperation project No.33-2, (ii) the National Science Foundation of China project No.70941035, and (iii) the Humanities and Social Sciences Research Program of Ministry of Education of the People's Republic of China, No.12YJA630161. Joanna Horabik acknowledges support from the Foundation for Polish Science under International $\mathrm{PhD}$ Projects in Intelligent Computing; a project financed by The European Union within the Innovative Economy Operational Programme 2007-2013 and European Regional Development Fund.

The authors thank the reviewers for their stimulating comments, which helped to shape the final version of the paper.

Open AccessThis article is distributed under the terms of the Creative Commons Attribution License which permits any use, distribution, and reproduction in any medium, provided the original author(s) and the source are credited.

\section{References}

ACM (2010) Approved consolidated baseline and monitoring methodology ACM0008. Consolidated methodology for coal bed methane, coal mine methane and ventilation air methane capture and use for power (electrical or motive) and heat and/or destruction through flaring or flameless oxidation. UNFCCC/CCNU CC. ACM0008/Version 07.Sectoral Scope: 08 and 10. EB 55. http://cdm.unfccc.int/methodologies

Bikhchandani S (1992) A bargaining model with incomplete information. J Econ Stud 59:187-203

Brown KA, MacCarthy J, Watterson JD, Thomas J (2010) Uncertainties in national inventory emissions of methane from landfills: A UK case study. In: Proceedings of the 3rd International Workshop on Uncertainty in Greenhouse Gas Inventories. Lviv Polytechnic National University, Lviv, pp 21-30

Hovi J (2001) Decentralized enforcement, sequential bargaining, and the clean development mechanism. CICERO Working Papers 12. https:/www.duo.uio.no/bitstream/handle/10852/32764/1617.pdf?sequence=1 Cited 6 Aug 2013.

IPCC (2007) IPCC fourth assessment report: climate change. http://www.ipcc.ch/publications_and_data/ publications_and_data_reports.shtml\#1

Kerachian R, Fallahnia M, Bazargan-Lari MR et al (2010) A fuzzy game theoretic approach for groundwater resources management: application of Rubinstein bargaining theory. Resour Conserv Recycl 54:673-682

Magalhães G, Espirito Santo Filho F, Alves JW, Kelson M, Moraes R (2010) Reducing the uncertainty of methane recovered $(\mathrm{R})$ in greenhouse gas inventories from waste sector and of adjustment factor (AF) in landfill gas projects under the clean development mechanism. In: Proceedings of the 3rd International Workshop on Uncertainty in Greenhouse Gas Inventories. Lviv Polytechnic National University, Lviv, pp 165-176 
Nahorski Z, Horabik J, Jonas M (2007) Compliance and emission trading under the Kyoto protocol: rules for uncertain inventories. Water Air Soil Pollut Focus 7(4-5):539-558

Nahorski Z, Stańczak J, Pałka P (2014) Simulation of an uncertain emission market for greenhouse gases using agent-based methods. Clim Chang. doi:10.1007/s10584-013-1039-2

Oh S-H, Heo G, Woo J-Ch (2010) Uncertainty of site-specific FOD for the national inventory of methane emission. In: Proceedings of the 3rd International Workshop on Uncertainty in Greenhouse Gas Inventories. Lviv Polytechnic National University, Lviv, pp 207-218

Project Document Description (2006) Huainan Panyi and Xieqiao coal mine methane utilization project. http:// www.dnv.com/focus/climate_change/Upload/PDD_Huainan $\% 20$ Panyi $\% 20$ and $\% 20 X i e q i a 0 \% 20 C M M$ 2006-01-01.pdf. Cited 10 Jan 2012

Rubinstein A (1982) Perfect equilibrium in a bargaining theory. Econometrica 50(1):97-109

Rubinstein A (1985) A bargaining model with incomplete information about time preferences. Econometrica 53(5):1151-1172

Shimada S, Funahashi Y, Li H et al (2005) Economic assessment of enhanced coal bed methane recovery in China. In: Proceedings of the 5th International Symposium on CBM/CMM in China. November 30December 12005:267-276

Srivastava J (2001) The role of inferences in sequential bargaining with one-sided incomplete information: some experimental evidence. Organ Behav Hum Decis 85(1):166-187

UNECE (2010) UNECE group of experts on coal mine methane. Comments on the ACM. http://cdm.unfccc.int/ methodologies/DB/OA37XAW7EI9WHJVZ97RGH2EZ5S9E93/view.html

UNFCCC (2011) Project cycle search database. http://cdm.unfccc.int/Projects/projsearch.html. Cited 1 March 2011

Utaki T (2010) Development of coal mine methane concentration technology for reduction of greenhouse gas emissions. Sci China Tech Sci 53(1):28-32

Vannetelbosch VJ (1997) Wage bargaining with incomplete information in an unionized Cournot oligopoly. Eur J Polit Econ 13:353-374

Xu X (2007a) Investigation in the Huainan coal mining area. Unpublished notes

$\mathrm{Xu}$ X (2007b) Risk analysis and control for potential coal bed methane CDM projects of China. China Min Mag 16:1-4 (in Chinese)

Zhang J, Wang H, Qian K et al (2004) Progress of CBM exploration research. China Coalbed Methane 1(1):1316 (in Chinese)

Zhang M, Ma G, Deyin Y et al (2005) Drainage technologies and utilization prospects of CMM in Hebi coal mining area. China Coalbed Methane 2(3):34-37 (in Chinese) 